\begin{tabular}{c} 
International Journal of Engineering \& Technology, $7(4.38)(2018)$ 1150-1153 \\
International Journal of Engineering \& Technology \\
SPC \\
Website: www.sciencepubco.com/index.php/IJET \\
Research paper \\
\hline
\end{tabular}

\title{
Analysis of thermomechanical self-oscillation In Perpendicular Free Cutting
}

\author{
Korendyasev G. K. \\ Institute of Machines Science named after A.A.Blagonravov of the Russian Academy of Sciences
}

\begin{abstract}
The results of the finite element modeling and analysis of thermomechanical phenomena arising from the of cutter self-excitation in perpendicular free cutting of metals are given. It is shown that the cause of self-excitation of self-oscillations is the nonlinear temperature dependence of the blank's mechanical characteristics. Modeling detected the effects of self-excitation and the establishment of selfoscillations, revealed the conditions for the propensity to the occurrence of self-oscillations for various materials.
\end{abstract}

Keywords: self-oscillations, thermomechanical model, drooping characteristic of cutting force, finite-element model.

\section{Introduction}

A thermomechanical model of self-excitations during cutting was proposed in [1]. The basis of this model is the assumption that the occurrence of self-oscillations arises because the mechanical characteristics of the material, its elastic modulus, yield point, and strength, and hence the cutting force depend on the temperature. Taking into account the reduction of cutting force with increasing temperature allows to open the relationship arising in the process of cutting, and turns cutter-blank system in a self-oscillating system with negative dissipation.

The thermomechanical model proposed in [1] gives a visual qualitative description and understanding of the phenomenon of the thermomechanical self-excitation. However, the prerequisites and assumptions adopted for its construction and solution, although based on experimental data, need clarification, taking into account the actual conditions of tool-blank contact and of heat transfer to the blank, cutter, chip, and the surroundings. This paper presents the results of finite element modeling of thermomechanical self-oscillations for perpendicular free cutting.

\subsection{Formation of a finite element model of a perpendicular free cutting process.}

The problem was solved in a two-dimensional formulation using the Abaqus Explicit (student edition) program based on explicit integration of the dynamic equations.

The initial data in the simulation are:

a) models of the blank and tool materials;

b) a model of the friction between the blank and tool;

c) a model of chip formation;

d) the geometry of the tool and blank

An ideal elastic plastic model of the material being processed was used in simulation. Data for the elastic modulus and yield point of the blank as a function of the temperature are borrowed from sources [2]. AISI 1045 steel was chosen as the material for the blank. The properties of this steel required for simulation depending on the temperature.
The tool is supposed to be absolutely rigid, therefore its material was described only by thermodynamic parameters and density. A hard alloy $\mathrm{H} 10 \mathrm{~F}$ was chosen as a tool material.

The process of friction of the tool's front surface with the chip and the friction of its rear surface with the blank is one of the insufficiently studied processes that accompany the cutting process.

The finite element simulation of cutting process is usually based on the Coulomb model of friction $\tau=\mu \sigma$, on account of its simplicity (where $\tau$ is the shear stress at contact; $\sigma$ is the normal stress at contact; and $\mu$ is the frictional coefficient). A priori selection of $\mu$ is the main source of error in determining the cutting force.

The treatment of chip separation is an important task. There are many numerical chip control algorithms. These algorithms can be divided into two main categories.

The first group is algorithms based on geometric assumptions. The essence of these algorithms is to create a geometric condition for the division of the grid points between the component and the chip. Due to the fact that the chip separation condition is chosen solely from geometrical considerations, this type of algorithm cannot reflect the real physics of the chip separation process and was used mainly in early works on finite element simulation of the cutting process [3].

The second group is the algorithms based on the physical criteria for the blank's failure. These criteria can be critical values of stresses, strains, and deformational energy. As a rule, these values can be gleaned from the test results of materials. These algorithms fairly reliably describe the physical processes occurring in the zone of chip formation. In the present work, the critical shear strain in the layer separating the blank and the chip is chosen as the criterion of failure.

In simulation, we adopt the following geometric parameters: cutting depth $2 \mathrm{~mm}$; cut width $3 \mathrm{~mm}$; tool's front angle $10^{\circ}$; tool's rear angle $5^{\circ}$.

We use four CPE4RT point elements on the basis of the "Distortion Control" option so as to retain grid quality with considerable plastic deformation of the blank. The grid becomes denser close to zones of expected high stress, strain, or 
temperature gradient. The size of the grid is selected to ensure sufficient precision but acceptable magnitude of the problem.

The boundary conditions of the model are shown in Figure 1. The blank moves at constant speed $V$ in the opposite direction to the tool. The tool with mass $m$ is connected to the immobile base by an elastically dissipative link with rigidity $c$ and viscous resistance $b$ and may move only along the $X$ axis. The initial conditions are a specified value of the system's initial temperature and velocity $V$ of the blank equal to the constant component of the cutting speed.

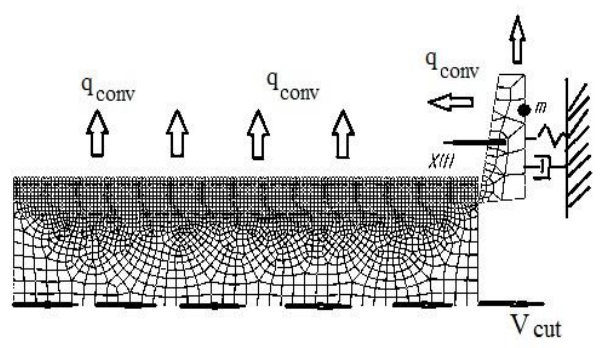

Figure 1: Boundary conditions of the model.

The maximum integration step is $\Delta t_{\max }=\min (L / c)$; where $\mathrm{L}$ is the characteristic dimension of a finite element, $c=\sqrt{E / \rho}$ is the longitudinal wave velocity in the blank.

\subsection{Analysis of thermomechanical oscillations of the cutter.}

The simulation results confirmed the possibility of exciting thermomechanical self-excitation. At certain ratios of the mechanical and thermal parameters of the blank, self-oscillations and their transition to steady-state periodic modes occur. Figure $2 \mathrm{a}$ shows a graph of temperature variation at tool - blank contact establishment of self - oscillation as the tool is inserted in the material.

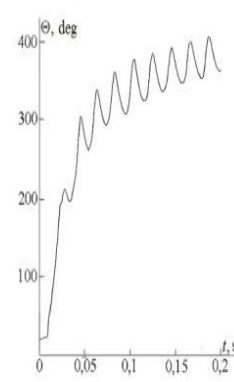

$a$

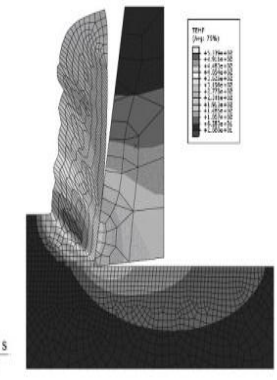

b

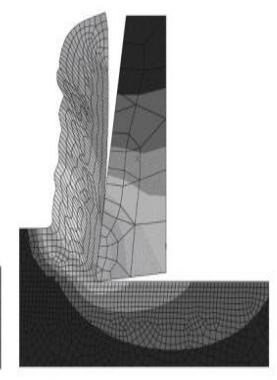

C
Figure 2: Graphs of temperature variations in the cutting zone.

It can be seen that at the beginning of the cutting process, the material is rapidly heated, and at temperature $\Theta \square \square \square \square \square \square$ selfoscillation is self-excited, and then further transition to the steadystate self-oscillation, accompanied by an increase in both the temperature average for the cycle and the amplitude of its cyclic. Analysis of the variation in contact temperature over time indicates a range of $50-60^{\circ} \mathrm{C}$ within one period of steady oscillation, the range may be $100^{\circ} \mathrm{C}$ in the shear zone. With an average temperature in the cutting zone equal to about $500^{\circ} \mathrm{C}$, the yield point in the shear zone varies by $10-15 \mathrm{MPa}$. These data give an idea of how powerful self-oscillations is the softening of the blank with increasing temperature in the cutting zone.

It should be noted that such significant temperature fluctuations occur in a fairly small volume. Figures $2 \mathrm{~b}$ and $2 \mathrm{c}$ show the patterns of temperature distribution in the cutting zone in two phases of the tool oscillations in the steady state at the instants when the temperature reaches the maximum (fig. $2 b$ ) and minimal (fig. $2 c$ ) values. In figure $2 b$, a local high-temperature region is clearly visible in the primary shear zone during the chip formation. Such localization and the presence of a boundary sharp temperature difference between this region and the surroundings was one of the main assumptions made in the construction of a mathematical model [1].

In simulation, graphs (Fig. 3) of changes in the basic parameters of the steady self-oscillation process as a function of time were obtained: 1 - the cutter displacement; 2 - the relative velocity of the cutter and the blank; 3 - the cutting force; 4 - the mean temperature at cutter - chip contact. For clarity, the presented curves are shown in normalized form.

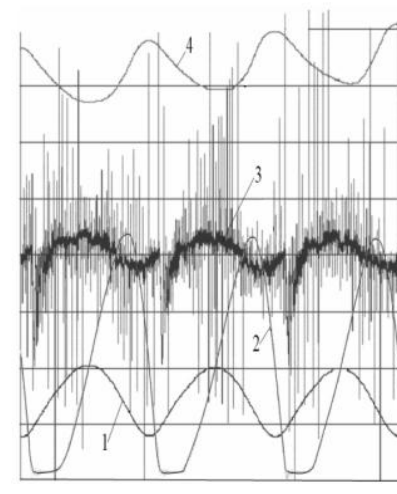

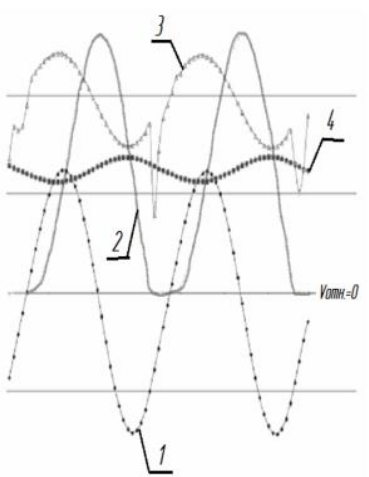

$b$
Figure 3: Graphs of changes in the system basic parameters

The oscillations of the cutter occur with the frequency of natural oscillations of an elastic system according to a law that is close to harmonic (curve 1). However, when moving in the same as the blank, the graph of the relative speed of the tool (curve 2) has a section where this speed is zero, i.e. the cutter is stationary relative to the blank and its speed in absolute motion is equal to the speed $V$ of the blank. During joint movement, the elastic cutter system stores energy from the blank's drive, and then. When the spring force is sufficient to overcome the resistance to cutting, the counter-movement of the cutter begins. From a comparison of the graph of relative speed with the graph of temperature change (curve 4), it can be seen that the temperature in the cutting zone decreases with passing movement and relative stop, and increases with the oncoming movement. Such temperature variation that creates the conditions for the onset and maintenance of selfoscillations.

Particular attention should be paid to the graph of the dependence of cutting force on time (curve 3 ). The cutting force tracks the change in temperature on average, but its graph contains additional high-frequency components. Apparently, they are a consequence of the discreteness of the model elements in the zone of the formation of chips. When the critical deformation is reached, the element is destroyed, which leads to an instantaneous change in the stresses in this zone, causing high-frequency oscillations. The correctness of this assumption is confirmed by the fact that the frequency of these high-frequency oscillations is approximately equal to the failure rate of finite elements on the contact line. Some contribution to the overall picture of the cutting force fluctuations is made by the elastic oscillations of the chips.

Additional confirmation of the above are the results (Fig. 3, b) obtained using the simplified model. In this model, it is assumed that the cutting force is created as a result of slip of a rectangular body over a plane, and the resistance to sliding force depends on temperature, following the laws of temperature dependence of the mechanical characteristics of the material being processed. The simplified model does not describe the chip formation process and this is mainly different from the one presented above. Comparison of the simulation results presented in Figure $3 \mathrm{a}, \mathrm{b}$ shows almost complete identity of the processes occurring in these systems, with 
the exception of the absence of high-frequency oscillations of the cutting force. It should be noted that the drop, seen in both systems, corresponds to elastic unloading in the cutting zone on switching from plastic deformation to an elastic stress state.

Self-oscillations very significantly affect the shape of the chip. Chips acquire a specific undultory form. Regions of significant plastic deformation when the tool and blank move in opposite directions alternate with regions of reduced deformation when the cutter moves away from the blank. The number of deformation waves at the chip corresponds to the number of oscillations. Figure 4 shows for comparison the diagrams of the distribution of equivalent plastic strains in chips during cutting under the conditions of presence (Fig. 4, a) and absence (Fig. 4, b) of selfoscillations.

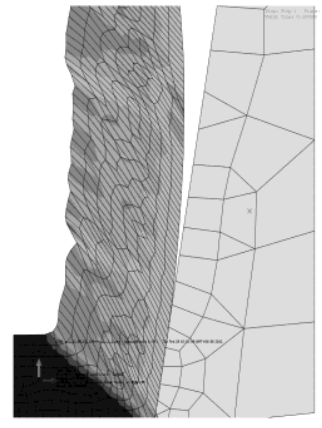

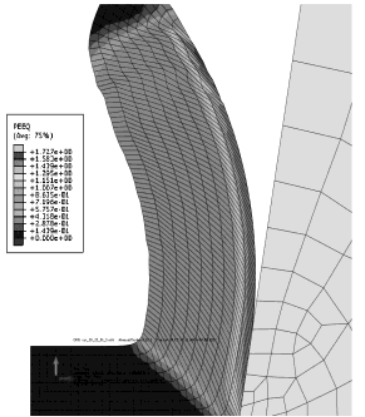

Figure 4: Plastic strains of chips.

To assess the effect of cutting speed on the nature of the selfoscillating process, a series of numerical experiments was carried out. Cutting speed varied from 0.05 to $3 \mathrm{~m} / \mathrm{s}$. According to the results of the experiments, it was found that the dependences of the self-oscillations amplitude $A$ on the cutting speed $V$ are extreme (Fig. 5), which is consistent with the experimental data [4]. The results of this study are very similar to the results of a similar study of a simplified model with adhesion.

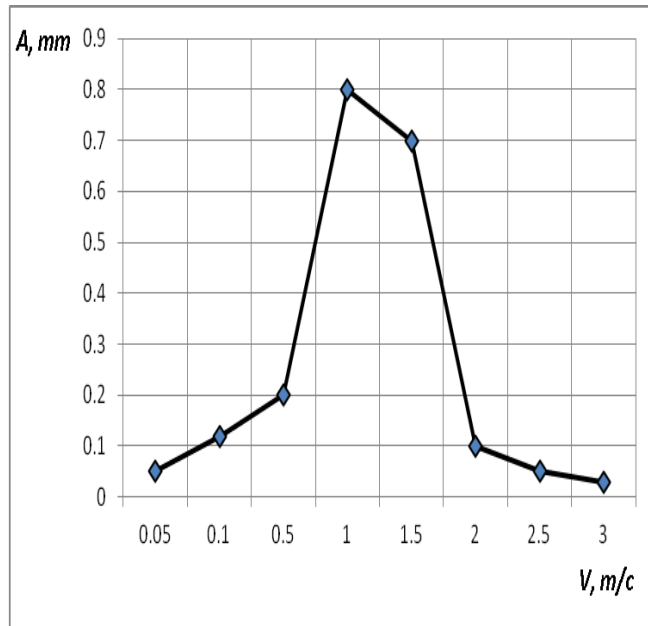

Figure 5: The dependences of the self-oscillations amplitude $A$ on the cutting speed $V$

\subsection{Analysis of the influence of the blank's properties on thermomechanical self-oscillations.}

This section presents the results of the analysis of the influence of the blank's properties on thermomechanical self-oscillations. A comparison was made of the behavior of the self-oscillatory system in the processing of steel, copper and aluminum. The mechanical and thermodynamic properties of these materials at different temperatures are given in $[3,5,6]$
The most important difference between these materials in terms of the effect on vibration during their processing is the difference in the values of the elastic modulus $E$ and the yield point $\sigma_{\mathrm{T}}$, which determines the magnitude of the cutting force.

Thus, with specified geometry of the system and specified properties of the tool a blank, the mean cutting force $P_{\mathrm{z}} 2500 \mathrm{~N}$, $1900 \mathrm{~N}$ for copper, $700 \mathrm{H}$ for aluminum. Obviously, with given tool rigidity, its deformations will be significantly different when cutting materials with such different values. The materials chosen by us have a pronounced falling characteristic $\sigma_{\mathrm{T}}=\sigma_{\mathrm{T}}(T)$, necessary for the self-excitation. Modeling shows that self-oscillations can be excited when processing all these materials in a wide range of cutting speeds. All other conditions being equal, the amplitude of self-oscillations has the following maximum values: $A_{\max }=0.8$ $\mathrm{mm}$ for steel; $A_{\max }=0.5 \mathrm{~mm}$ for copper; $A_{\max }=0.2 \mathrm{~mm}$ for aluminum. The simulation was carried out with the same cutting geometry.

The comparison of the thermal field arising from the processing of various materials under conditions of thermomechanical selfoscillations is of interest. In figure $6 \mathrm{a}, \mathrm{b}$, c, we show the temperature fields for steel, copper and aluminum. The reason for such a different nature of the temperature distribution is the difference in the thermodynamic parameters of the selected materials, primarily thermal conductivities. As can be seen from the figure, the heating of the blank is greatest for aluminum, which has a very high thermal conductivity. The heating is least for steel, with low coefficient of thermal conductivity.

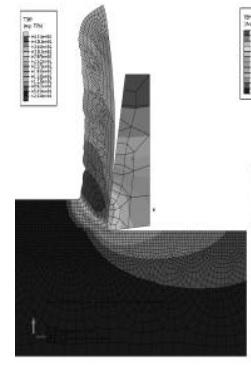

a) steel

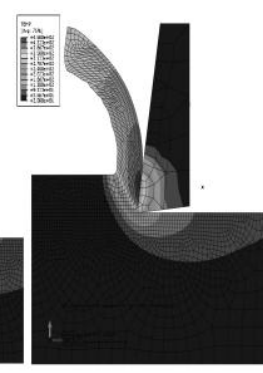

b)copper

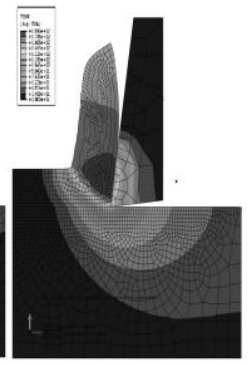

c) aluminum
Figure 6: Comparison of thermal fields in the processing of various metals.

Reducing the heat capacity, as well as increasing thermal conductivity improves the conditions of heat removal from the cutting zone. When the cutter moves from the blank in steady selfoscillations, the shear region has time to cool. This creates favorable conditions for maintaining self-oscillations.

However, as mentioned above, the decisive factor determining the vibration resistance of the system is the values of the yield point $\sigma_{\mathrm{T}}$ and elastic modulus $E$ and their temperature variation.

\section{The main results of the study.}

Investigation of finite-element model of cutting tool selfoscillations provided checking the correctness of an assumption about thermomechanical nature of self-oscillations during the cutting and adequacy of this process mathematical model. The type of obtained results is in agreement with results of natural experiments [4]. Created models can serve for finding cutting modes, providing minimal level of vibrations, as well as during designing metal-cutting tools and furniture. Understanding of physical mechanism of self-oscillations excitation as an interdependent thermomechanical process allows making clear physical sense of phenomena, being fundamental for the most commonly encountered models of self-oscillations during the cutting [7-9]. At present time an experimental work is carried out on checkout of adequacy for the thermomechanical model of selfoscillation excitation during the cutting, consisting in full-scale study of vibratory displacement fields, temperature fields and deformation fields emerging during self-oscillations. 


\section{References:}

[1] Astashev, V.K. and Korendyasev, G.K., Thermomechanical Model of the Occurrence of Oscillations in Metal Cutting, ISSN 1052 6188, Journal of Machinery Manufacture and Reliability, 2012, Vol. 41, No. 3, pp. 189-193. (C) Allerton Press, Inc., 2012

[2] http://www.matweb.com.

[3] Komvopoulos, K. and Erpenbeck, S.A., Finite Element Modeling of Orthogonal Metal Cutting, Trans. ASME J. Eng. Ind., 1991, vol. 113 , pp. 253-267.

[4] Zharkov, I.G., Vibratsii pri obrabotke lezviinym instrumentom [Vibration in Tool Cutting], Leningrad: Mashinostroenie, 1987.

[5] Kesharwani, R., High-Temperature Behavior of Copper, Master's Thesis, National Institute of Technology, Orissa, India, 2010.

[6] http://www.engineeringtoolbox.com.

[7] Kashirin, A.I., Issledovarie vibratsii pri rezanii metallov [Vibrations in Metal Cutting], Moscow: Izd. AN SSSR, 1944.

[8] Sokolovskii, A.P., Vibratsii pri rabote na metallorezhushchikh stankakh: Issledovanie kolebanii metallorezhushchikh stankov pri rezanii metallov [Vibration in Metal-Cutting Machines: Oscillations in Metal Cutting], Moscow: Mashgiz, 1958.

[9] El'yasberg, M.E., Osnovi teorii avtokolebaniy pri rezanii metallov Self- Theory of Oscillation of Metal-Cutting Machines// Stanki i instrument 1962. № 10, №11. 